\title{
Tomato SIRUP is a negative regulator of UV-B photomorphogenesis
}

\author{
Chunli Zhang ${ }^{1,2}$, Qianwen Zhang ${ }^{1,2}$, Hongye Guo ${ }^{1,2}$, Xiaohui Yu ${ }^{3}$, Wenjing Liang ${ }^{1,2}$, Yinhua Chen ${ }^{3}$, Ruohe Yin ${ }^{1,2}$ and \\ Li $\operatorname{Lin}^{1,2^{*}}$ (D)
}

\section{Introduction}

Sunlight is an environmental factor regulating plant growth and development. Plants have evolved a battery of photoreceptors to sense and respond to different wavelengths of light (Jenkins, 2017; Liu et al., 2020a). UV-B (280-315 nm) comprises the shortest wavelength of sunlight on the earth surface. In general, low fluence rate UV-B does not generate damage effects on plants, rather it induces plant photomorphogenic responses (Yin and Ulm, 2017). High fluence rate UV-B may damage macromolecules, including nucleic acids, protein and membranes (Hideg et al., 2013). The UV-B photoreceptor UVR8 protein ((UV RESISTANCE LOCUS 8) perceives the low dose UV-B and triggers UV-B specific signal transduction. The photomorphogenic responses mediated by UVR8 play a key role in UV-B acclimation and plant survival in nature (Favory et al., 2009). UVR8 exists as homodimer in its ground state and UV-B perception leads to dimer dissociation into monomer, which is the active form for signaling (Rizzini et al., 2011). In Arabidopsis, RUP1 (REPRESSORS OF UV PHOTOMORPHOGENESIS 1) and RUP2 are two key negative regulators of UV-B signaling (Gruber et al., 2010). RUP1 and RUP2 promote the reversion of UVR8 monomer to homodimer (Heijde and Ulm, 2013).

Tomato (Solanum lycopersicum) is an agriculturally and economically important vegetable crop. In agriculture, tomato seedlings may face sudden UV-B exposure when transferred from seedling bed in green house,

\footnotetext{
* Correspondence: li.lin@sjtu.edu.cn

${ }^{1}$ School of Agriculture and Biology, Shanghai Jiao Tong University, 800 Dongchuan RD, Minhang District, Shanghai 200240, People's Republic of China

${ }^{2}$ Key Laboratory of Urban Agriculture (Ministry of Agriculture) and Joint Center for Single Cell Biology, School of Agriculture and Biology, Shanghai Jiao Tong University, Shanghai 200240, People's Republic of China Full list of author information is available at the end of the article
}

where UV-B is blocked by plastic cover or glass, to open field. Therefore, it is important to investigate how tomato seedlings respond to UV-B. Previously, we demonstrated that Tomato UVR8 (SIUVR8) regulates seedling photomorphogenesis and UV-B stress tolerance (Liu et al., 2020b). However, the negative regulation of UV-B signaling is not known.

\section{Results}

In Arabidopsis, the two highly related WD40 proteins RUP1 and RUP2 (AtRUP1 and AtRUP2) were identified to be repressors of UVR8-mediated UV-B signaling pathway (Gruber et al., 2010). We found that AtRUP1 and AtRUP2 show high identity to only one putative protein encoded by Solyc11g005190 in Tomato genome (55.5 and 56.1\%, respectively). The Solyc11g005190 was therefore called tomato RUP (SIRUP). Previously, SIRUP was shown to be a negative regulator of photomorphogenesis in white light, where it was named as LeCOP1Like (Liu et al., 2004). For clarity we renamed LeCOP1Like as SIRUP herein. Like AtRUP1 and AtRUP2, SIRUP mainly consists of WD40-repeats (TrpAsp) domain with apparently no additional domains (Fig. S1A\&B). Transcript of SlRUP was induced by UV-B in a SlUVR8- and SlHY5-dependent manner (Fig. 1A).

To analyze the physiological functions of SIRUP, we generated SIRUP overexpression lines with C-terminal GFP, and Slrup mutants by CRISPR/CAS9 approach (Fig. S2). Three independent SIRUP-GFP overexpression lines were selected by immunoblot analysis (Fig.S2A). Two homozygous Slrup mutant lines were identified by PCR-resequencing, with $111 \mathrm{bp}$ and $113 \mathrm{bp}$ deletion, respectively (Fig.S2B-E). Under white light, Slrup mutants developed shorter hypocotyls than wildtype (AC, Ailsa Craig) (Fig. 1B\&C). UV-B can inhibit hypocotyl elongation of seedlings, the hypocotyl length of wildtype 

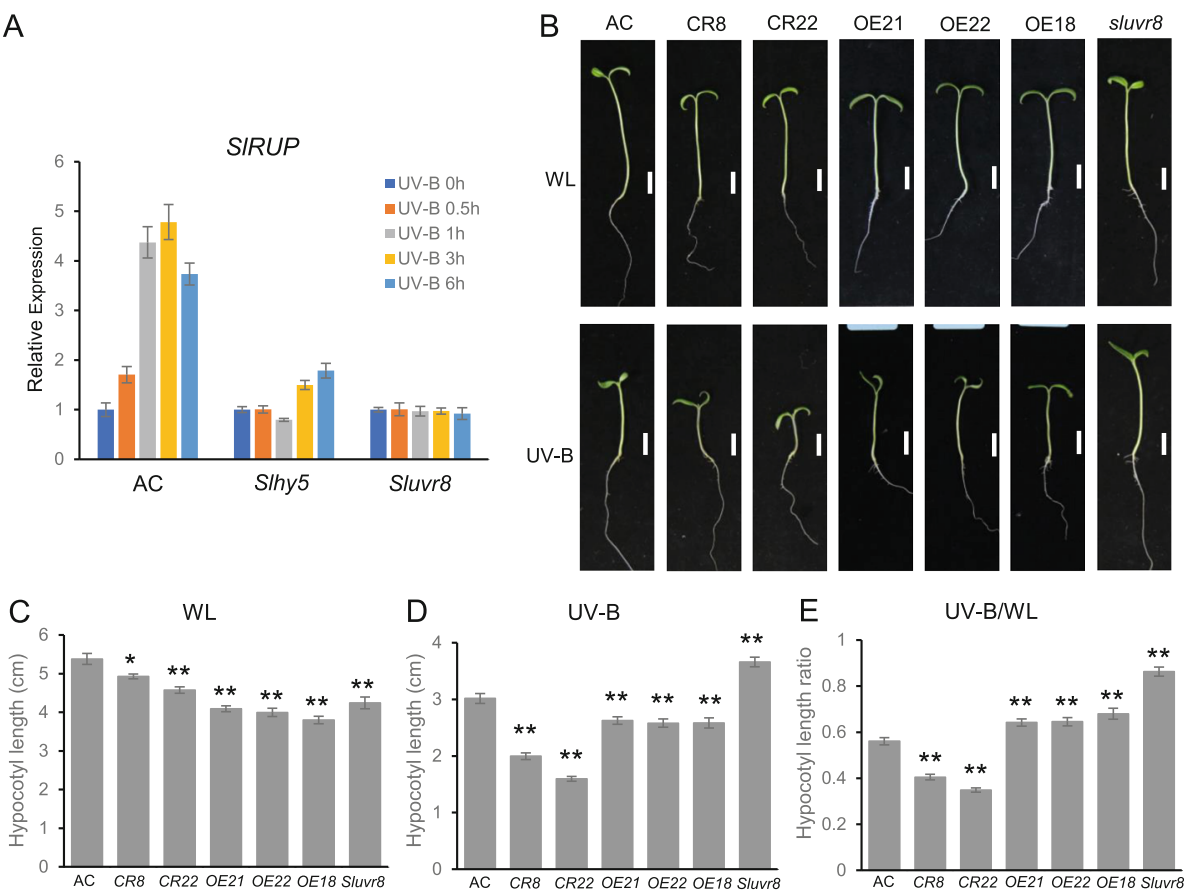

D UV-B

E

UV-B/WL
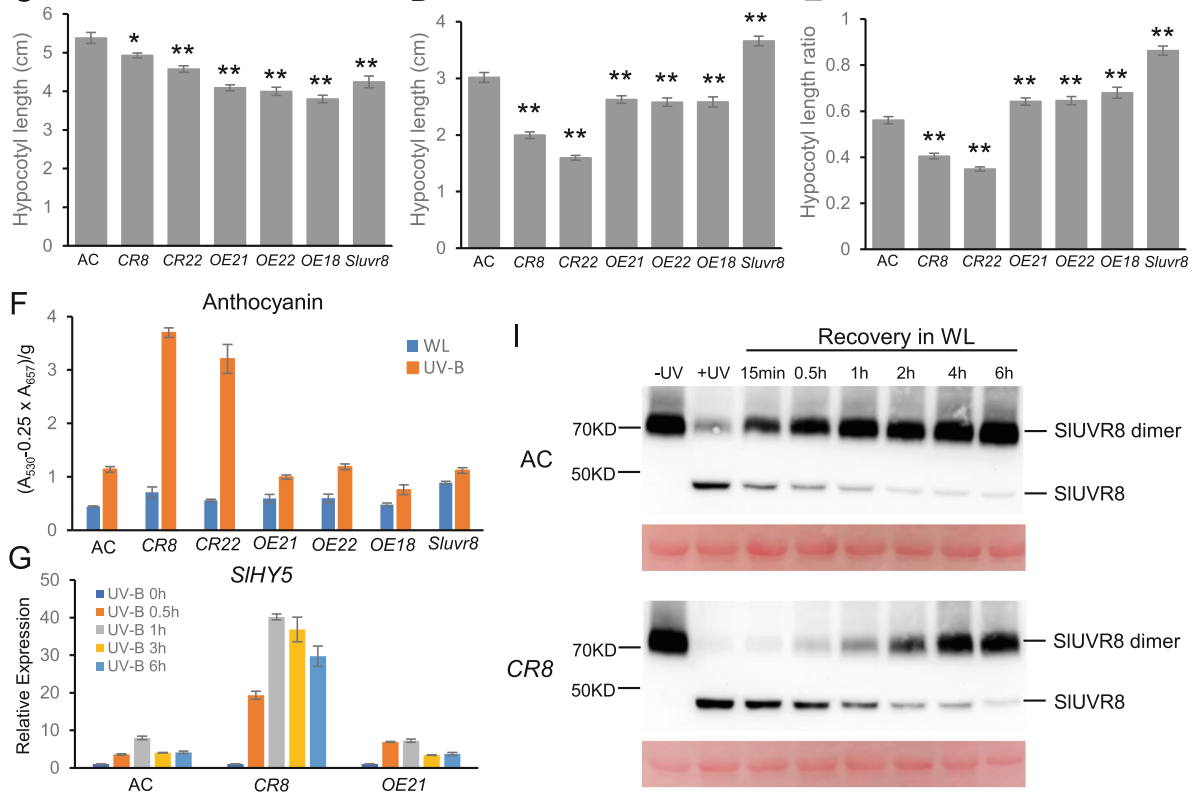
AC

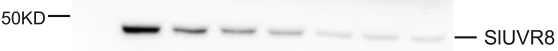

CR8
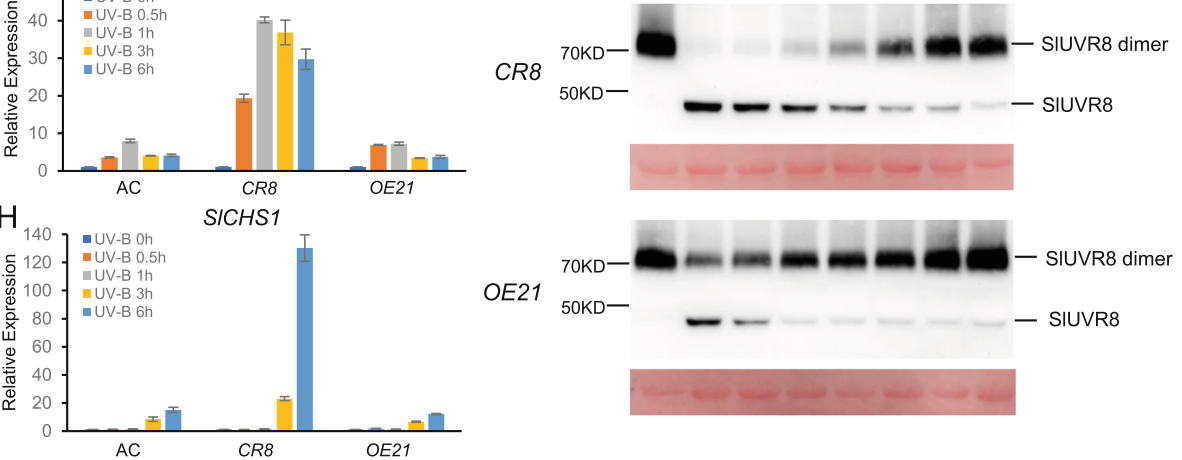

Fig. 1 SIRUP negatively regulates the UVR8-mediated UV-B photomorphogenesis. (A) Transcript analysis of SIRUP in response to UV-B in Sluvr8, Slhy5 mutants compared with wildtype (AC, Ailsa Craig) by qRT-PCR. 6d old seedlings were irradiated with UV-B for different lengths. Mean and SE of three biological replicates are presented. (B) Representative seedlings of wildtype and Slrup mutants (CR8 and CR22), and SIRUP-GFP lines grown in white light or white light supplemented with UV-B. Bar $=1 \mathrm{~cm}$. (C) and (D) Hypocotyl length of $6 \mathrm{~d}$ old seedlings grown under white light (WL) or white light supplemented with UV-B (UV-B), Mean and SE are presented $(n=30)$. (E) The hypocotyl length ratio of UV-B/WL. Asterisks indicate significant differences between transgenic lines and wildtype, differences with a $P<0.05\left({ }^{*}\right)$ and $P<0.01\left(*^{*}\right)$ resulting from a one-way analysis of variance test. (F) UV-B induced anthocyanin accumulation. Mean and SE are presented $(n=3)$. (G) and $(H)$ qRT-PCR analysis of SIHY5 and SICHS1 in response to UV-B in wildtype, Slrup mutant, and SIRUP-GFP line for different UV-B radiation time. Mean and SE of three biological samples are presented. (I) SIRUP promotes redimeriation of SIUVR8 post UV-B treatment. $6 \mathrm{~d}$ old seedlings were irradiated with broadband UV-B for $0.5 \mathrm{~h}$, followed by recovery in white light (WL) in the absence of UV-B for different time. Anti-SIUVR8 was used as the primary antibody for immunoblot analysis. Ponceau staining serves as loading control 
seedling under UV-B was only about $56 \%$ of that under white light (Fig. 1D\&E). Slrup mutants and RUP-GFP overexpression seedlings were hypersensitive and hyposensitive to UV-B, respectively (Fig. 1B, D, E). Consistent with the key function of SIUVR8 in UV-B signaling, hypocotyl length of Sluvr8 mutant was only slightly shortened under UV-B in comparison to that under white light. Relative to white light control, UV-B induced anthocyanin content to about 3 folds in wildtype, 6-7 folds in Slrup mutants, and only 1.5-2 folds in SIRUPGFP overexpression lines (Fig. 1F). HY5 and CHS1 play key roles in anthocyanin biosynthesis. Transcription of both SlHY5 and SlCHS1 was induced by UV-B much more pronounced in Slrup than in wildtype and SIRUPGFP lines (Fig. 1G \& H, Fig. S3). Intriguingly, the UV-Binduced expression of SlHY5 and SlCHS was similar in wildtype and SIRUP-GFP overexpression lines. Based on those observations, we conclude that SIRUP is a negative regulator of UVR8-mediated UV-B photomorphogenesis.

We tested possible mechanism for SIRUP in UV-B signaling. SlUVR8 protein levels were similar in Slrup mutant and wildtype (Fig. S4), suggesting that SIRUP does not regulate SIUVR8 protein levels. In response to UVB, UVR8 homodimers dissociate rapidly to monomers, which trigger UV-B signaling in plants (Rizzini et al., 2011). We tested whether SIRUP regulates the dimer/ monomer conformational change of SIUVR8. Under white light, SIUVR8 was detected as homodimer and UV-B induced the dissociation of homodimer into monomer in wildtype, the Slrup mutant and the SIRUPGFP overexpression line. In wildtype seedling, SIUVR8 showed dimer recovery initiated within $15 \mathrm{~min}$, and completed within $2 \mathrm{~h}$ post UV-B exposure. The rate of SIUVR8 redimerization in the Slrup mutant was slow and monomer was detectable even $4 \mathrm{~h}$ post UV-B (Fig. 1I, Fig. S5A). SlUVR8 redimeration appears to be slightly faster in SIRUP-GFP overexpression line than wildtype post UV-B (Fig. 1I, Fig. S5B). Thus, we conclude that SIRUP accelerates redimeration of UV-B-activated SIUVR8 monomer.

To survive in nature, plants need to acquire mechanisms to cope with high dose UV-B stress. We previously showed that the acclimation to high dose UV-B stress was dependent on photoreceptor SIUVR8 (Liu et al., 2020b). We tested whether SIRUP participates in UV-B stress tolerance. Exposure of seedlings to stress UV-B (broadband) caused seriously stress response in wildtype, SIRUP-GFP, and Sluvr8 mutant seedlings, whereas, Slrup mutant was obviously more resistant (Fig. S6). Consistent with the previous report, a beforehand exposure of seedlings to photomorphogenic UV-B (acclimated) for $2 \mathrm{~d}$ enhanced tolerance to broadband UV-B stress in wildtype, but not in Sluvr8 mutant (Fig. S6) (Liu et al., 2020b). Interestingly, this acclimation effect was attenuated in SlRUP-GFP lines and enhanced in Slrup mutants (Fig. S6). This result demonstrated that SIRUP could negatively regulate UV-B acclimation and tolerance to UV-B stress of tomato seedlings.

\section{Discussion}

In agricultural, tomato seedlings may face sudden UV-B exposure at seedling stage when transferred from UV-Bshielded green house to UV-B-rich open field. Thus, the understanding of UV-B responses of tomato seedlings is important for better agriculture practice. In this work, we characterized a key negative regulator of UV-B signaling pathway in tomato. Slrup mutants were hypersensitive to low fluence rate UV-B in the assays tested including hypocotyl elongation, UV-B target gene expression and anthocyanin accumulation. Through comparison of SIUVR8 photocycle in wildtype, Slrup mutant and SIRUP overexpression line, it is evident that SIRUP inhibits UV-B signaling via promoting the reversion of active SIUVR8 monomer to inactive homodimer. This mechanism is well conserved in Arabidopsis (Heijde and Ulm, 2013). Previous study showed that pigments and carotenoids are altered in SIRUP-RNAi tomato fruits (Liu et al., 2004). Further work is needed to investigate how SIRUP regulates tomato fruit metabolisms.

In a previous report, the SIRUP-RNAi line developed shorter hypocotyl and dark green leaves than wildtype in white light and dark (Liu et al., 2004). Thus, SIRUP is a negative regulator of photomorphogenesis not only in UV-B but also in white light. Intriguingly, overexpression of SIRUP also leads to shorter hypocotyls in white light. Thus, balanced expression of RUP is required for proper seedling photomorphogenesis. The mechanism for the function of SIRUP in white light awaits further investigation.

In summary, this study revealed that SIRUP is a key repressor of SIUVR8-mediated UV-B photomorphogenesis in tomato. SIRUP inhibits UV-B signaling via accelerating the conversion of active SIUVR8 monomer to inactive homodimer. In addition, SIRUP negatively regulates UV-B stress tolerance of tomato seedlings, which is important for agriculture practice.

\section{Supplementary Information}

The online version contains supplementary material available at https://doi. org/10.1186/s43897-021-00010-z.

\section{Additional file 1. Materials and Methods. Table S1: Primers used in this} work

Additional file 2 Fig. S1 Structural conservation of Tomato RUP. (A) Amino acid sequences alignment of SIRUP, AtRUP1, and AtRUP2. (B) Schematic representation of the protein domain structures of SIRUP, AtRUP1 and AtRUP2. WD40 represents WD40-repeats (Trp-Asp) domain. Fig. S2 Generation of SIrup mutants and SIRUP-GFP overexpression lines. (A) Immunoblotting analysis with SIRUP-GFP overexpression lines. 
An anti-GFP antibody was used for immunoblotting. Ponceau Staining serves as loading control. (B) Schematic illustration of the two sgRNAs target sites on the SIRUP genomic sequence, blue box represents exon. Two sgRNAs targeting to the SIRUP coding sequence are in red font, and PAM (protospacer adjacent motif) in gray. (C) Sequence-based genotyping of CRISPR/Cas9-SIRUP homozygous mutant. The target sites are underlined and the PAM are highlighted in gray. The deletions are indicated by dashes. (D) and (E) Amino acid sequences alignment of SIRUP wildtype, Slrup-CR8 (C) and Slrup-CR22 (D) mutant. Fig. S3 Transcript expression of SIHY5 and SICHS1 in response to UV-B in SIRUP transgenic lines. qRT-PCR analysis of SIHY5 (A) and SICHS1 (B) in response to UV-B in wildtype (AC, Ailsa Craig), Slrup mutant, and SIRUP-GFP lines for different UV-B radiation time. Mean and SE of three biological samples are presented. Fig. S4 SIUVR8 protein levels in AC and Slrup mutant in white light and white light with supplemental UV-B. $6 \mathrm{~d}$ old tomato seedlings were either grown in white light (-UV) or white light supplemented with UV-B (+UV) for $2 \mathrm{~h}$. An anti-SIUVR8 antibody was used for immunoblot analysis. Ponceau Staining serves as loading control. Fig. S5 SIRUP promotes redimerization of SIUVR8. $6 \mathrm{~d}$ old seedlings of AC (Ailsa (raig), SIrup mutant and SIRUP overexpression seedlings were irradiated with broadband UV-B for $0.5 \mathrm{~h}$, followed by recovery in white light (WL) for different time. Anti-SIUVR8 antibody was used as the primary antibody for immunoblot analysis. Heat-denatured protein samples demonstrated total amounts of SIUVR8 protein. Ponceau Staining serves as loading control. Fig. S6. SIRUP negatively regulates UV-B acclimation and tolerance. Tomato seedlings were grown for $7 \mathrm{~d}$ under white light $\left(20 \mathrm{\mu mol} \cdot \mathrm{m}^{-2} \cdot \mathrm{s}^{-1}\right)$, then seedlings were treated with white light supplemented with photomorphogenic UV-B (Philips TL20W/01RS, $1.5 \mu \mathrm{mol} \cdot \mathrm{m}^{-}$ ${ }^{2} \cdot s^{-1}$ ) for $2 \mathrm{~d}$ (acclimated) or white light for $2 \mathrm{~d}$ (control and nonacclimated). Seedlings were then irradiated with broadband UV-B for $1 \mathrm{~h}$ (non-acclimated and acclimated), or subjected to a $1 \mathrm{~h}$ mock treatment (control) under white light. Treated seedlings were further grown for $4 \mathrm{~d}$ under white light without UV-B before being photographed. (A) Representative seedlings of AC (Ailsa Craig), Slrup mutants, and SIRUP-GFP lines recovery in white light without UV-B for $4 \mathrm{~d}$. (B) Mean and SE of seedling fresh weight were shown $(n=9)$.

\section{Acknowledgements}

We thank Xiaorui Liu for initial cloning of SIRUP.

\section{Authors' contributions}

C.Z. performed experiments, and prepared initial draft of the manuscript, Q.Z. and W.L. participated s/rup mutants and overexpression lines confirmation. H.G., X.Y and Y.C contributed analytic tools. R.Y. analyzed data and revised the manuscript. L.L. designed the project, analyzed data and revised the manuscript. The authors read and approved the final manuscript.

\section{Funding}

This work was supported by the National Natural Science Foundation of China (31870261 \& 32070261), Natural Science Foundation of Shanghai (18ZR1419600), the Agri-X Interdisciplinary Fund of Shanghai Jiao Tong University (Agri-X2017006).

\section{Availability of data and materials}

The author responsible for distribution of materials integral to the findings presented in this article in accordance with the policy described in the Instructions for Authors in Molecular Horticulture is: ruohe.yin@sjtu.edu.cn

\section{Declarations}

Ethics approval and consent to participate

Not applicable.

\section{Consent for publication}

All the authors have agreed publication of this manuscript.

\section{Competing interests}

The authors declare that they have no conflict of interest.

\section{Author details}

School of Agriculture and Biology, Shanghai Jiao Tong University, 800 Dongchuan RD, Minhang District, Shanghai 200240, People's Republic of China. ${ }^{2}$ Key Laboratory of Urban Agriculture (Ministry of Agriculture) and Joint Center for Single Cell Biology, School of Agriculture and Biology, Shanghai Jiao Tong University, Shanghai 200240, People's Republic of China. ${ }^{3}$ Hainan Key Laboratory for Sustainable Utilization of Tropical Bioresource, Hainan University, Haikou 570228, People's Republic of China.

Received: 8 February 2021 Accepted: 12 April 2021

Published online: 27 August 2021

References

Favory JJ, Stec A, Gruber H, Rizzini L, Oravecz A, Funk M, et al. Interaction of COP1 and UVR8 regulates UV-B-induced photomorphogenesis and stress acclimation in Arabidopsis. EMBO J. 2009;28(5):591-601. https://doi.org/10.1 038/emboj.2009.4.

Gruber H, Heijde M, Heller W, Albert A, Seidlitz HK, Ulm R. Negative feedback regulation of UV-B-induced photomorphogenesis and stress acclimation in Arabidopsis. Proc Natl Acad Sci U S A. 2010;107(46):20132-7. https://doi.org/1 $0.1073 /$ pnas. 0914532107.

Heijde M, Ulm R. Reversion of the Arabidopsis UV-B photoreceptor UVR8 to the homodimeric ground state. Proc Natl Acad Sci U S A. 2013;110(3):1113-8. https://doi.org/10.1073/pnas.1214237110.

Hideg E, Jansen MA, Strid A. UV-B exposure, ROS, and stress: inseparable companions or loosely linked associates? Trends Plant Sci. 2013;18(2):107-15. https://doi.org/10.1016/j.tplants.2012.09.003.

Jenkins GI. Photomorphogenic responses to ultraviolet-B light. Plant Cell Environ. 2017:40(11):2544-57. https://doi.org/10.1111/pce.12934.

Liu H, Lin R, Deng XW. Photobiology: light signal transduction and photomorphogenesis. J Integr Plant Biol. 2020a;62(9):1267-9. https://doi. org/10.1111/jipb.13004.

Liu X, Zhang Q, Yang G, Zhang C, Dong H, Liu Y, et al. Pivotal roles of tomato photoreceptor SIUVR8 in seedling development and UV-B stress tolerance. Biochem Biophys Res Commun. 2020b;522(1):177-83. https://doi.org/10.1016/ j.bbrc.2019.11.073.

Liu Y, Roof S, Ye Z, Barry C, van Tuinen A, Vrebalov J, et al. Manipulation of light signal transduction as a means of modifying fruit nutritional quality in tomato. Proc Natl Acad Sci U S A. 2004;101(26):9897-902. https://doi.org/10.1 073/pnas.0400935101.

Rizzini L, Favory JJ, Cloix C, Faggionato D, O'Hara A, Kaiserli E, et al. Perception of UV-B by the Arabidopsis UVR8 protein. Science. 2011;332(6025):103-6. https://doi.org/10.1126/science.1200660.

Yin R, Ulm R. How plants cope with UV-B: from perception to response. Curr Opin Plant Biol. 2017;37:42-8. https://doi.org/10.1016/j.pbi.2017.03.013.

\section{Publisher's Note}

Springer Nature remains neutral with regard to jurisdictional claims in published maps and institutional affiliations.

Ready to submit your research? Choose BMC and benefit from:

- fast, convenient online submission

- thorough peer review by experienced researchers in your field

- rapid publication on acceptance

- support for research data, including large and complex data types

- gold Open Access which fosters wider collaboration and increased citations

- maximum visibility for your research: over $100 \mathrm{M}$ website views per year

At BMC, research is always in progress.

Learn more biomedcentral.com/submission 